Recepción: 10 / 04 / 2018

Aceptación: 27 / 06 / 2018

Publicación: 15 / 09 / 2018

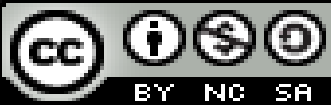

Ciencias de la educación

Artículo de investigación

\title{
Resistencia a los cambios curriculares en la educación a distancia
}

Resistance to curricular changes in distance education

Resistência às mudanças curriculares na educação à distância

Maria E. Salazar-Quevedo I

mariaedith32@hotmail.com

\section{Correspondencia: mariaedith32@hotmail.com}

${ }^{\text {I }}$ Magister en Educación a Distancia, Licenciada en Administración Turística, Tecnóloga en Administración Turística, Docente de la Universidad Nacional de Loja, Loja, Ecuador. 


\title{
Resumen
}

El presente estudio tuvo como propósito evaluar la resistencia a los cambios curriculares en estudiantes de la educación a distancia. La investigación se ubicó en un estudio descriptivo y de campo. La población fue de 65 estudiantes de todas las carreras durante el segundo semestre del año 2017. La técnica utilizada para la recolección de los datos fue la encuesta a través de un instrumento tipo cuestionario con tres alternativas de respuestas: siempre, algunas veces y nunca. La validez del instrumento fue de contenido por criterios de expertos en el área y la confiabilidad se determinó a través del Coeficiente de Alfa Cronbach, cuyo resultado fue de 0.91, considerado altamente significativo. La técnica de análisis fue la estadística descriptiva e incluye una relación chi cuadrado. Entre los resultados se evidenció que existe una relación altamente significativa de dependencia entre los resultados se evidenció que solo algunas veces se dan las resistencias lógicas, psicológicas y sociológicas en los estudiantes de educación a distancia.

Palabras claves: educación a distancia; cambios curriculares; resistencia lógica; resistencia psicológica y resistencia sociológica.

\begin{abstract}
Purpose of this study was to evaluate the resistance to curricular changes in distance education students. The research was a descriptive study and field. The population was 65 students of all races during the second half of the year 2017. The technique used for the collection of data was the survey through a questionnaire type instrument with three alternative answers: sometimes always, and never. The validity of the instrument was content by experts in the field criteria and reliability was determined by the Cronbach Alpha coefficient, whose result was 0.91, considered highly significant. The technique of analysis was descriptive statistics and includes a chi square relationship. The results evidenced that there is a highly significant relationship of dependence between the results evidenced that only sometimes logical, psychological and sociological resistances in education students are given distance.
\end{abstract}

Keywords: distance education; curricular changes; logical strength; psychological resistance and resistance sociological. 


\section{Resumo}

O objetivo deste estudo foi avaliar a resistência a mudanças curriculares em estudantes de educação a distância. A investigação foi localizada em um estudo descritivo e de campo. A população foi de 65 alunos de todas as carreiras durante o segundo semestre de 2017. A técnica utilizada para coleta dos dados foi a pesquisa por meio de um instrumento do tipo questionário com três respostas alternativas: sempre, às vezes e nunca. A validade do instrumento foi de conteúdo por critério perito na área e a confiabilidade foi determinada pelo coeficiente de Alpha Cronbach, cujo resultado foi de 0,91 , considerado altamente significativo. A técnica de análise foi estatística descritiva e inclui uma relação qui-quadrado. Entre os resultados constatou-se que existe uma relação de dependência altamente significativa entre os resultados, que mostrou que apenas algumas vezes ocorrem resistências lógicas, psicológicas e sociológicas nos alunos de educação a distância.

Palavras chave: educação a distância; mudanças curriculares; resistência lógica; resistência psicológica e resistência sociológica.

\section{Desarrollo}

Herrera y López (1995) indican que, "la interacción constructiva que se establece entre la comunidad y la universidad se podrá aprovechar hasta lograr constituirlas como una comunidad única y cohesionada" (pag.26). Por tanto, se debe estimular al estudiante a negociar e intercambiar ideas para unificar esfuerzos en la aplicación de proyectos y entender que su participación en el proceso se considera vital para que se logren los objetivos de la institución. En atención a esta posición, es notorio que todo conocimiento implica cambios del cual suelen surgir resistencia y obstáculos en materia educativa. A tal efecto, en este artículo se evalúa la resistencia a los cambios curriculares en estudiantes de la educación a distancia.

\section{Resistencia a los Cambios Educativos}

La resistencia al cambio educativo está en todos los niveles de la organización, desde el estudiante hasta los docentes. El estudiante en las instituciones educativas tiende a resistir el cambio aun cuando éste traiga consigo nuevas experiencias. Para Robbins (2006) la resistencia al 
cambio puede ser una fuente de conflicto funcional. El cambio puede convertirse en un éxito o un problema según la habilidad con que se administre para aminorar en lo posible la resistencia.

Según Chiavenato (2009) considera, que todo dentro de una resistencia es rutinario, estandarizado y previsto con anticipación, el funcionario se acostumbra generalmente a una completa estabilidad y repetición de aquello que hace, lo que le brinda total seguridad acerca de su futuro en la burocracia, atendiendo a las normas y reglamentos impuestos por ésta; el funcionario se vuelve simplemente un ejecutor de rutinas y procedimientos, los cuales pasa a dominar con plena seguridad y tranquilidad con el correr del tiempo.

En efecto, cuando surgen los cambios dentro de las instituciones, el estudiante los interpreta como algo que él desconoce y, por lo tanto, algo que puede poner en peligro su seguridad y tranquilidad; con esto el cambio pasa a ser indeseable para el estudiante, quien en la medida de lo posible se resistirá a cualquier tipo de innovación que quiera implementarse en la institución. Tal resistencia puede ser tanto pasiva como activa y agresiva, manifestándose en comportamientos de reclamo y agitación.

Según Davis y Newstrom (2001) "existen tres clases de resistencia que pueden expresarse con los términos: lógica, psicológica y sociológica" (pag.56). El autor manifiesta, que la resistencia lógica tiene como base el pensamiento racional ya que el alumno se resiste a aceptar cualquier tipo de cambio e innovación que pone en peligro su estabilidad y equidad.

La resistencia psicológica es propia del ser humano que se vincula con las emociones, actitudes y sentimientos individuales del docente, ya que es una conducta inconsciente del individuo, lo que lo lleva a decir no como parte de su proceso de vivir. La resistencia sociológica es asociada a los intereses y valores del grupo y es una fuerza poderosa en el ambiente laboral y que debe ser atendida con mucho cuidado.

Resistencia Lógica. En este contexto, la resistencia lógica tiene base en el pensamiento racional y científico y surge del tiempo y del esfuerzo que se requiere para ajustarse al cambio, incluye las labores que deben emprenderse o se emprenden en la organización, esto representa costos que deben aportar las organizaciones. Aun cuando a la larga el cambio puede ser favorable para la 
organización, los costos a corto plazo deben pagarse primero, pero solo con bienes tangibles en términos de productividad.

Al respecto, Carrasco (2007) se refiere, a la resistencia lógica, "al conocimiento humano que ayuda a discernir y separa los contenidos

esenciales de los secundarios, a descubrir las relaciones que existen

entre ellos" (pag.45). De esto se infiere, que la resistencia lógica es propia del ser humano y que esta le ayuda a separar el pensamiento racional del emocional como sentimiento, actitudes y emociones que el autor llama secundarios. El pensamiento racional que el autor plantea, como contenidos esenciales propios del conocimiento humano que al descubrirlos pone al descubierto la relación que existe entre ellos.

Según Chiavenato (2009) "todo cambio exige ciertos ajustes personales y modificaciones que ocurren a través de modos diferentes: en los patrones de comportamiento" (pag.87). En el primer modo los ajustes comportamentales se refieren a las alteraciones objetivas que deben hacerse por aquellos que efectúan el trabajo mediante rutinas físicas en la ejecución de tareas. Así, la lógica derrumba los patrones de comportamiento, que deben adaptarse a nuevos reglamentos, procedimientos y métodos de trabajo. Y, en consecuencia, deben alcanzarse nuevos patrones de comunicación, cooperación e interacción.

Resistencia Psicológica. Los ya citado ajustes o modificaciones emergen para vencer resistencias que en el orden psicológico se vincula con las emociones, los sentimientos y las actitudes en términos de sentimientos individuales de los estudiantes con respecto al cambio, genera desconfianza del liderazgo de la gerencia, pueden sentir amenaza o inseguridad. Es evidente, que aun cuando la gerencia considere que no existe justificación de esos sentimientos, éstos son reales y deben considerarse para reorientarlos.

Al respecto, Chiavenato (2009) plantea, que "la resistencia psicológica sigue un patrón de actitudes personales" (pag.98). Es decir, la resistencia psicológica en las instituciones educativas se manifiesta en las actitudes desarrolladas por las personas con relación al cambio en función de su habilidad para afrontar las nuevas exigencias. En efecto, si un participante o estudiante percibe 
que es capaz de ajustarse al cambio sin grandes sacrificios personales, y si percibe que el resultado final del cambio le es bastante benéfico, se dice que adopta una actitud psicológicamente positiva al cambio, lo que representa una fortaleza para la organización académica. Según Latner (2006) plantea, los orígenes de la resistencia se encuentran en la imposición del propio ego sobre la actividad cotidiana del quehacer del hombre, lo que hace que éste pierda la pista de lo que se esté haciendo, a fin de alcanzar con éxito sus necesidades.

Las resistencias como conducta inconsciente mantienen al hombre del presente y, por lo tanto, les generan dificultades para afrontar nuevas necesidades. Lo que hace necesario, es que se traiga a la conciencia, el contacto con la resistencia; de tal manera, que el acto de decir no, se considere como parte del proceso de vivir. Al mismo tiempo, se puede escoger y probar alguna otra forma de conducción o por el contrario, seguir la misma. El mismo autor, señala que la resistencia tal como se emplea en psicología, tiene algunas implicaciones que no forman parte del enfoque Gestalt. La resistencia no solamente son rasgos indeseables o neuróticos; más bien son gestaltes formados en circunstancias apremiantes. Según los resultados de la formación anterior de la figura son partes integrantes del funcionamiento personal para ayudar al organismo a enfrentar situaciones, que según el individuo es la más adecuada.

En la terapia Gestalt las resistencias son el foco central del trabajo terapéutico puesto que son los puntos en que el paciente demuestra su interferencia con el funcionamiento libre; y al mismo tiempo estos puntos presentan aspectos de si mismo que son básicos para él. El paciente está actuando tal como es. Las resistencias son oportunidades para aumentar la conciencia del paciente e incluir en ella la incapacidad y la falta de deseos de experimentar algo. Desde este punto de vista, la terapia Gestalt se puede entender como un examen continuo y desarrollo de las resistencias.

Resistencia Sociológica. La resistencia sociológica se asocia con los intereses y los valores del grupo y asume un carácter lógico en términos de los intereses y valores de la organización. La resistencia sociológica en las instituciones educativas se entiende como una fuerza poderosa del ambiente a la que debe atender con todo cuidado, coaliciones políticas, valores opuestos de los estudiantes y juicios distintos de las comunidades educativas. En un grupo pequeño existen amistades de trabajo que se perturban con el cambio. Los estudiantes necesitan hacer que las 
condiciones del cambio sean lo más favorable posible para mejorar con éxito las resistencias sociológicas.

Según Chiavenato (2009) "la resistencia sociológica viene dada en la adaptación social del individuo" (pag.67). En efecto, si la persona se siente insegura e incapaz y no percibe ningún beneficio personal proveniente del cambio, su actitud personal puede ser negativa y de oposición a éste. De este modo, la resistencia sociológica es generada por un proceso de adaptación social que al verse afectado comienza a generarse en él, un sentimiento de inseguridad e incapacidad al observar que el cambio planteado no le genera ninguna satisfacción personal y comienza a generarse en este una oposición negativa a la innovación o transformación planteada. En un plano educativo, Carrasco (2007) plantea que:

La resistencia sociológica permite determinar cuáles son aquellos conocimientos, habilidades, normas y valores que es preciso poner al alcance de los estudiantes, para que los hagan suyos en su momento y sean miembros activos y responsables de la institución a la que pertenecen. (pag.122).

Evidentemente, estos tipos de resistencia deben manejarse con eficiencia, si se espera que los estudiantes cooperen con su propio cambio. Si los estudiantes se preocupan solamente por los aspectos técnicos y lógicos del cambio, tienden a fracasar en su responsabilidad social y humana. El manejo de la resistencia en el ámbito educativo es de fundamental importancia, ya que ésta puede convertirse en una fuerza poderosa que se debe administrar con la mejor habilidad y eficiencia para lograr que las condiciones del cambio sean lo más favorables posibles para así superar con éxito las resistencias y lograr la mayor productividad de los estudiantes en el proceso de cambio y la adquisición de nuevas experiencias se conviertan en un éxito.

\section{El Cambio del Sistema de Evaluación y el Cambio de Mentalidad}

También se aprecia la resistencia al cambio en la práctica docente vinculada al nuevo reglamento de evaluación. De este modo, dicha práctica docente habitual, han distorsionado muchos de los principios de la evaluación, plasmados en la Normativa Vigente. En opinión, de muchos especialistas del país, el reglamento actual de evaluación posee valiosos aportes para una adecuada evaluación de los aprendizajes. Sin embargo, este marco legal de la evaluación 
educativa, se fundamenta en la constatación de logros de objetivos, de acuerdo con una pormenorización de taxonomías de verbos, enfoque que según las investigaciones realizadas en el campo del aprendizaje de las ciencias se encuentra muy cuestionado.

En efecto, se está frente a un nuevo paradigma educativo, que ha generado una abundante investigación, producida durante las últimas décadas, y arrojando buenos resultados en el aprendizaje. Puede suponerse que el diseño curricular del sistema de Educación a Distancia, debe tomar en cuenta estos avances y ser acompañado con un cambio similar en la evaluación. Una vez que se han producido cambios importantes en el conocimiento de cómo la gente aprende, y esta base teórica orienta el diseño de nuevas metodologías, la evaluación debe ser coherente con las nuevas propuestas. Estos cambios no son fáciles de asimilar, sobre todo, cuando la cultura evaluativa instaurada se fundamenta en constatar aciertos y errores para clasificar a los sujetos en exitosos y fracasados.

Cuando se ha insistido en castigar los errores y los alumnos han sido condicionados a esconderlos con refuerzos negativos, se hace difícil, tanto para el docente como para el estudiante tratar de explicar su error, para hacer reflexiones y crecer a partir de él. La evaluación en último caso estará dirigida a valorar los esfuerzos que ha realizado el estudiante para superar su equivocación y su capacidad para plantearse nuevos retos de aprendizaje.

Al respecto, en la cultura de educación la evaluación de la práctica docente juega un papel importante. De modo, que no se evalúa, pero si se califica. Esta distinción es de vital importancia pues muchas veces el bajo rendimiento, tiene su origen en el reemplazo de la evaluación por la calificación. La calificación es un acto administrativo de control académico respecto al alumno y la evaluación juzga el rendimiento de ese alumno para mejorarlo.

Asimismo, Vilchez (2001) expresa, "que el rendimiento de los estudiantes suministra la data fundamental que activa y desata cualquier proceso evaluativo" (pag.33). Es decir, se evalúa al presumir que la enseñanza aprendizaje es un proceso imperfecto (de naturaleza humana). El rendimiento de los estudiantes, el desempeño docente e incluso la idoneidad de los programas interesan esta vez como un dato colectivo. Es evidente, que la información es valiosa para cada alumno y cada docente en particular, solo que desde la perspectiva curricular es válido el 
comportamiento colectivo y las tendencias masivas. Como dato relevante para la evaluación curricular, las informaciones que provienen del desempeño de los alumnos y de los docentes deben ser generalizadas e involucrar al conjunto como una totalidad.

\section{Metodología}

La investigación se ubicó en un estudio de tipo descriptivo de campo, de acuerdo a Hernández, Fernández y Baptista (2010), afirman que la investigación descriptiva es la que se orienta a redactar informes relacionados con el estado real de las personas, objeto situaciones o fenómenos tal cual se presentan en el momento de su recolección, describe lo que mide sin analizar inferencias ni verificar hipótesis. La población la constituyeron 65 estudiantes del sistema de educación a distancia durante el segundo semestre del año 2017. La técnica utilizada para la recolección de los datos fue la encuesta a través de un instrumento tipo cuestionario con tres alternativas de respuestas: siempre, algunas veces y nunca. La validez del instrumento fue realizada a través del criterio de expertos en el área y la confiabilidad se determinó a través del Coeficiente de Alfa Cronbach, cuyo resultado fue de 0.91, considerado altamente significativo. La técnica de análisis fue la estadística descriptiva.

\section{Resultados}

En este aparte se presentan los resultados del análisis estadístico que permitió evaluar la resistencia a los cambios curriculares en estudiantes de la educación a distancia, los mismos se presentan en tablas con sus respectivos análisis.

Tabla 1. Distribución frecuencial y porcentual del indicador resistencia lógica

\begin{tabular}{ccccc}
\hline \hline Alternativas/AnÁlisis & FR & \% & FRA & \%A \\
\hline \hline Siempre & 10 & 15 & 10 & 15 \\
\hline AlgunAs VeCES & 35 & 54 & 45 & 69 \\
\hline NunCA & 20 & 31 & 65 & 100 \\
\hline TOtaL & $\mathbf{6 5}$ & $\mathbf{1 0 0}$ & $\mathbf{6 5}$ & $\mathbf{1 0 0}$ \\
\hline \hline
\end{tabular}

Fuente: Elaboración propia 
En la tabla 1, se observa la distribución frecuencial y porcentual del indicador resistencia lógica, en la que se observa que el $54 \%$ del total de los estudiantes investigados opinó que algunas veces necesitan estar totalmente motivados para poder entender que forma parte de un programa de estudios a distancia que exige mucho tiempo y esfuerzo para ajustarse o relacionarse con los módulos de estudio. Seguido del 31\% que señaló la alternativa nunca. El menor porcentaje se indicó para estudiantes que se inclinaron por la alternativa siempre con el 15\% del total de las opiniones.

Tabla 2. Distribución frecuencial y porcentual del indicador resistencia psicológica

\begin{tabular}{ccccc}
\hline \hline \multirow{2}{*}{ Alternativas/AnÁlisis } & FR & \% & FRA & \%A \\
\hline \hline Siempre & 11 & 17 & 11 & 17 \\
\hline ALGUNAS VeCES & 32 & 49 & 43 & 66 \\
\hline NunCA & 22 & 34 & 65 & 100 \\
\hline TOtAL & $\mathbf{6 5}$ & $\mathbf{1 0 0}$ & $\mathbf{6 5}$ & $\mathbf{1 0 0}$ \\
\hline \hline
\end{tabular}

Fuente: Elaboración propia

En la tabla 2, se observa la distribución frecuencial y porcentual del indicador resistencia psicológica, en la que se observa que el $49 \%$ del total de los estudiantes investigados opinó que algunas veces sienten inseguridad de la carrera que estudian y necesitan de grandes sacrificios personales para ajustarse a los esfuerzos que exigen sus estudios. Seguido del 34\% que respondió nunca. El menor porcentaje fue para estudiantes que se inclinaron por la alternativa siempre con el 17\% del total de las opiniones.

Tabla 3. Distribución frecuencial y porcentual del indicador resistencia sociológica

\begin{tabular}{ccccc}
\hline \hline Alternativas/AnÁLISIS & FR & \% & FRA & \%A \\
\hline \hline SiEMPRE & 9 & 14 & 9 & 14 \\
\hline ALGUNAS VeCES & 37 & 57 & 46 & 71 \\
\hline NUNCA & 19 & 29 & 65 & 100 \\
\hline TOTAL & $\mathbf{6 5}$ & $\mathbf{1 0 0}$ & $\mathbf{6 5}$ & $\mathbf{1 0 0}$ \\
\hline \hline
\end{tabular}

Fuente: Elaboración propia 
En la tabla 3, se presenta la distribución frecuencial y porcentual del indicador resistencia sociológica, en el que se observa que el $57 \%$ del total de los estudiantes investigados opinó que algunas veces se sienten seguros y adaptados socialmente en su sistema de estudios y atienden con cuidado las influencias del medio ambiente como las coaliciones políticas, valores opuestos entre los estudiantes y juicios distintos entre los compañeros de estudios. Seguido del 29\% que señaló la alternativa nunca. El menor porcentaje se indicó para estudiantes que se inclinaron por la opción siempre con el $14 \%$ del total de las opiniones.

\section{Conclusiones}

La resistencia lógica está presente en los estudiantes algunas veces, como propia del ser humano y que todo cambio exige ciertos ajustes para adaptarse y lograr con éxito los objetivos propuestos.

Algunas veces, la resistencia sociológica en los estudiantes se vincula con sus emociones individuales, lo que les genera inseguridad o incertidumbre en el transcurso de su carrera elegida, lo que implica mayor esfuerzo y cambio de aptitudes y actitudes, para poder desarrollar habilidades que le permitan dominar su desempeño académico.

La resistencia psicológica, algunas veces en opinión de los estudiantes, lo consideran fundamental en este tipo de sistema, ya que su actitud puede desarrollar una posición negativa o positiva, en cuanto a su entorno institucional y pudiera convertirse en una fuerza poderosa para alcanzar con éxito el cambio de actitud y lograr la mayor productividad frente al sistema de estudios no presencial.

\section{Referencias bibliográficas}

Carrasco (2007). Los cambios en el proyecto no nos competen. Recuperado en: https://www.eluniverso.com/2007/.../F86B6014E18141B8A2579FBE6F531DA7.html

Chiavenato (2009). Administración de Recursos Humanos, 5ta. Ed. McGraw. Hill, Colombia.

Davis y Newstrom (2001). Comportamiento humano en el trabajo. Recuperado en: https://www.urbe.edu/UDWLibrary/InfoBook.do?id=9725 
Hernández R, Fernández C y Baptista P (2010). Metodología de la Investigación. Tercera Edición. Editorial Mc Graw Hill. México.

Herrera y López (1995). Educación para superar la pobreza y alcanzar la equidad - Ucab. Recuperado en: https://www.ucab.edu.ve/.../INV-IIES-REV-081-Educacion-para-superar-lapobreza

Latner (2006). A Guide to Assessments That Work. Recuperado en: https://books.google.co.ve/books?isbn=0190671963

Robbins E (2006). Comportamiento Organizacional Controversias y Aplicaciones. Editorial McGraw Hill. México.

Vílchez (2001). Análisis de la gestión de la Plataforma de Aprendizaje Virtual. Recuperado en: https://www.researchgate.net/.../28153017 